\title{
Search for SUSY with a Highly Compressed Mass Spectrum in the Soft Single Lepton Channel with the CMS Experiment
}

\author{
Mateusz Zarucki*i \\ HEPHY \\ E-mail: mateusz.zarucki@cern.ch
}

\begin{abstract}
SUSY models with compressed mass spectra are highly motivated by theoretical considerations, such as dark matter constraints and naturalness. The presented search targets simplified models of SUSY in which the signal consists of top squark (stop) pair production, followed by four-body decays or decays via an intermediate chargino. The models assume the neutralino to be the lightest supersymmetric particle (LSP), with a mass gap between the stop and the LSP that is smaller than the $\mathrm{W}$ boson mass. The difficulty with identifying the resulting soft decay products can be mitigated by requiring the presence of an initial-state radiation jet, which boosts the system. The data is analysed using two approaches: a sequential selection based on discriminating variables and a multivariate technique. The results are based on a sample of proton-proton data from Run II of the Large Hadron Collider, recorded with the CMS detector at a centre-of-mass energy of $13 \mathrm{TeV}$, corresponding to an integrated luminosity of $35.9 \mathrm{fb}^{-1}$. No significant deviation from the standard model is observed and upper limits are set on the stop production cross section in the plane of the top squark vs. LSP masses, excluding stop masses up to $560 \mathrm{GeV}$. Furthermore, a statistical combination with the results of an all-hadronic channel is presented, yielding the most stringent limits in this region of the SUSY parameter space with 2016 data.
\end{abstract}

An Alpine LHC Physics Summit (ALPS2018)

15-20 April, 2018

Obergurgl, Austria

${ }^{*}$ Speaker.

${ }^{\dagger}$ on behalf of the CMS Collaboration 


\section{Introduction}

Supersymmetry (SUSY) is one of the most promising theories that could provide solutions to central open questions and problems in the standard model (SM). SUSY postulates that for every SM particle there exists a corresponding superpartner state, differing by half-integer spin. The additional particle content predicted by SUSY would provide a solution to the hierarchy or naturalness problem, while minimal supersymmetric extensions of the SM (MSSM) could allow for the unification of strong and electroweak gauge couplings at the GUT scale. Furthermore, the lightest supersymmetric particle (LSP), which is weakly-interacting, massive and stable, is an excellent dark matter (DM) candidate. A focus of the physics programme at the Large Hadron Collider (LHC), CERN, is physics beyond the standard model (BSM), which includes searches for SUSY. Many SUSY models require R-parity conservation, meaning that the SUSY particles are pair produced and are predicted to have decay chains to SM particles, which can be detected with the Compact Muon Solenoid (CMS) detector [1].

Wide regions of the SUSY parameter space have been excluded with recent Run II results, however, there exist 'crevasses' of compressed mass parameter space that have not yet been probed. The models in these regions are characterised by mass splittings $\Delta m$ between the next-to-LSP and LSP that are comparably small. There are additional theoretical motivations for SUSY models with such compressed spectra, for example, the prediction that the process of co-annihilation of light stops and LSPs could reproduce the correct cosmological DM relic density [2].

The analysis [3, 4] presented in this paper focuses on this compressed region of SUSY parameter space. The simplified models used for optimising and interpreting the analysis constitute the pair production of the top squarks (stops), which are the superpartners of the top quark, followed by either direct decays of the stop to a four-body final state or via an intermediate chargino $\left(\tilde{\chi}_{1}^{ \pm}\right)$. The process is shown in Figure 1 and it is considered decoupled from the full SUSY theory, since other SUSY particles do not contribute to the process. The lightest neutralino $\left(\tilde{\chi}_{1}^{0}\right)$ is considered the LSP, while the only free parameters are the stop and LSP masses. A specific compressed scenario is investigated where the mass difference between the stop and LSP $\Delta m=m_{\mathfrak{t}}-m_{\mathrm{LSP}}<m_{\mathrm{W}}$; here $m_{\mathrm{W}}$ is the mass of the $\mathrm{W}$ boson and thus the stop decays via an off-shell top $t^{*}$ and off-shell $W^{*}$. This results in low momenta of the visible decay products, which typically do not pass detector acceptance thresholds. The LSPs and the neutrino escape the detector, leading to a missing transverse momentum $p_{\mathrm{T}}^{\text {miss }}$ signature, which is also small in magnitude. In the presence of initial state radiation (ISR), the system is boosted and the visible decay products become detectable, while the $p_{\mathrm{T}}^{\text {miss }}$ increases. Therefore, the targeted signature contains a high momentum ISR jet, moderate $p_{\mathrm{T}}^{\text {miss }}$ and a low momentum lepton, as the analysis targets the single-lepton final state.

\section{Samples and Event Selection}

The sample of events under consideration come from proton-proton (pp) data from Run II of the Large Hadron Collider, recorded with the CMS detector at a centre-of-mass energy $\sqrt{s}=13 \mathrm{TeV}$, corresponding to an integrated luminosity of $35.9 \mathrm{fb}^{-1}$. Simulated Monte Carlo (MC) samples of SM background and signal events are produced using several generators and reconstructed with the same algorithms as data. 

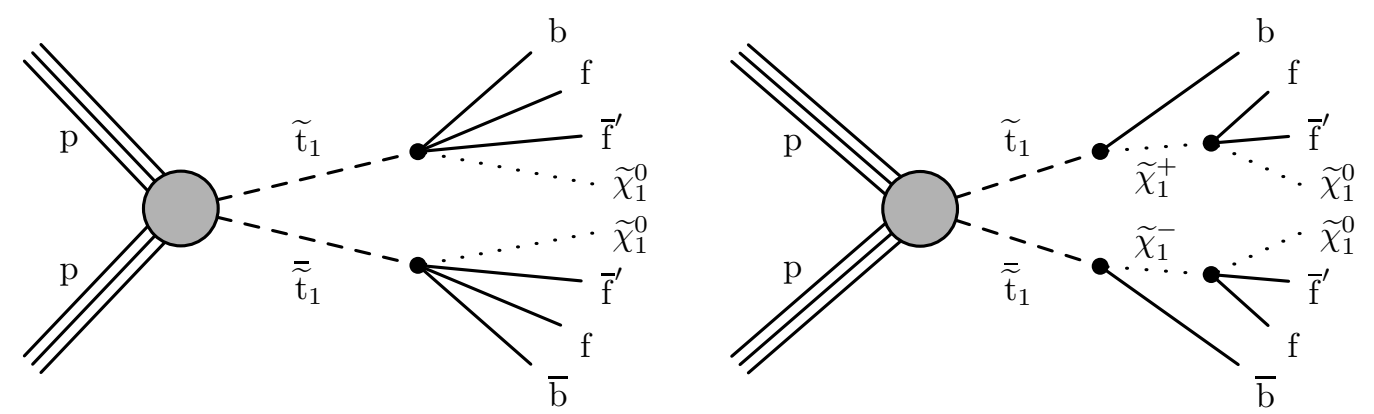

Figure 1: Simplified signal models for top squark pair production with subsequent four-body (left) and chargino-mediated (right) decays into a lepton-neutrino (quark-antiquark) pair, b-quark jet and the LSP. The chargino $\left(\tilde{\chi}_{1}^{ \pm}\right)$mass is assumed to be halfway between the stop and LSP [3].

The data is analysed using two approaches: a sequential selection based on discriminating variables, cut \& count $(\mathrm{C} \& \mathrm{C})$, and a multivariate analysis technique (MVA). Both methods rely on the same baseline event selection with only a few minor differences that are used to maximise the sensitivity. The events are required to have been triggered by an online selection on $p_{\mathrm{T}}^{\text {miss }}$ and preselection criteria are applied to focus on the relevant parameter space. These include kinematic requirements on the missing momentum $p_{\mathrm{T}}^{\text {miss }}>200(280) \mathrm{GeV}$ and hadronic energy $H_{\mathrm{T}}>300(200) \mathrm{GeV}$ for the C\&C (MVA) approach. The latter variable is defined as the scalar sum of the $p_{\mathrm{T}}$ of all jets with $p_{\mathrm{T}}>30 \mathrm{GeV}$ and pseudorapidity $|\eta|<2.4$. We require the leading jet in the event to have $p_{\mathrm{T}}>100(110) \mathrm{GeV}$ for the C\&C (MVA) analysis, so it is compatible with ISR. Even though the QCD-multijet events are dominant processes in pp collisions, in this analysis they are already significantly reduced with the requirement of relatively high $p_{\mathrm{T}}^{\text {miss }}$. Additional criteria are applied to further reduce the remaining QCD background. Since it predominantly has a back-to-back topology, events are required to have at most two jets with $p_{\mathrm{T}}>60 \mathrm{GeV}$ and an angle $\Delta \phi<2.5 \mathrm{rad}$ between them. Concerning the leptonic selection, at least one muon with $p_{\mathrm{T}}>3.5 \mathrm{GeV}$ and $|\eta|<2.4$ or an electron $p_{\mathrm{T}}>5 \mathrm{GeV}$ and $|\eta|<2.5$ is required. Reconstructed taus and additional light leptons with $p_{\mathrm{T}}>20 \mathrm{GeV}$ are vetoed. The distributions of the key variables used in the analysis, the lepton $p_{\mathrm{T}}$ and transverse mass $m_{\mathrm{T}}$, are shown in Figure 2 at the level of the $\mathrm{C} \& \mathrm{C}$ preselection.

\subsection{Cut \& Count $(\mathrm{C} \& C)$ Method}

In the C\&C approach, two sets of signal regions (SRs) are chosen, SR1 and SR2, targeting a range of values of $\Delta m$ to enhance the signal-to-background ratio. The leptons are required to have $p_{\mathrm{T}}<30 \mathrm{GeV}$ and for SR1 also $|\eta|<1.5$. SR1 targets very low $\Delta m$ 's where the b jets are expected to be too soft to be detected, and are therefore vetoed, whereas SR2 requires at least a soft $\mathrm{b}$ jet with $30<p_{\mathrm{T}}<60 \mathrm{GeV}$, targeting higher mass splittings. To further increase the sensitivity of the analysis, both SRs are binned in lepton $p_{\mathrm{T}}(\mathrm{L}: 5-12 \mathrm{GeV}, \mathrm{M}: 12-20 \mathrm{GeV}$ and $\mathrm{H}: 20-30 \mathrm{GeV}$ ). In the muon channel, there is an additional very low (VL) bin with $3.5<p_{\mathrm{T}}<5 \mathrm{GeV}$. The transverse mass $m_{\mathrm{T}}$ is calculated between the lepton $p_{\mathrm{T}}$ and $p_{\mathrm{T}}^{\text {miss }}: m_{\mathrm{T}} \equiv \sqrt{2 p_{\mathrm{T}}^{\text {miss }} p_{\mathrm{T}}(1-\cos (\Delta \Phi))}$. It has a characteristic shape for both $W+$ jets and $\bar{t}$, which are the dominant backgrounds of the analysis, 

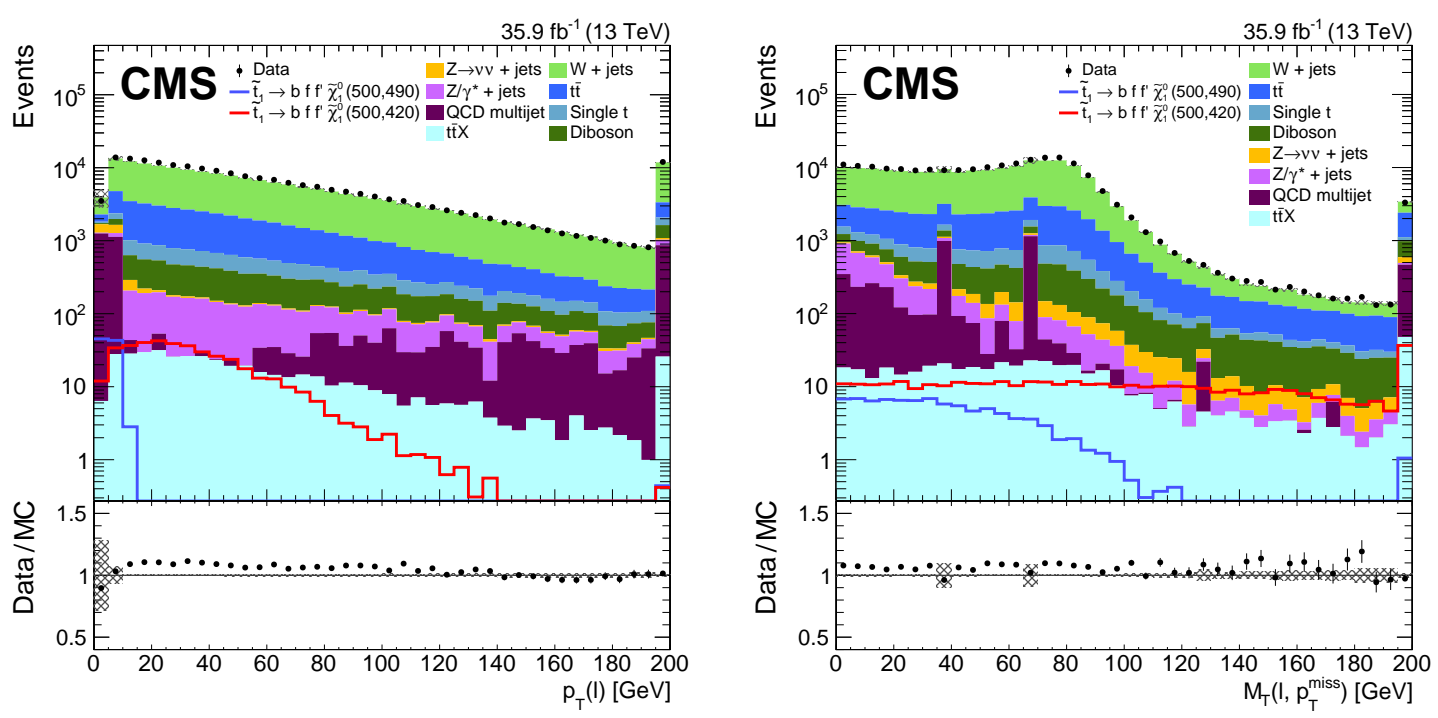

Figure 2: Distributions of the lepton $p_{\mathrm{T}}$ (left) and transverse mass $m_{\mathrm{T}}$ (right) at the level of preselection [3] ${ }^{1}$.

forming a peak around the $\mathrm{W}$ boson mass. A splitting in $m_{\mathrm{T}}$ (a: $m_{\mathrm{T}}<60 \mathrm{GeV}$, b: $60<m_{\mathrm{T}}<95 \mathrm{GeV}$ and c: $m_{\mathrm{T}}>95 \mathrm{GeV}$ ) is done to isolate this $\mathrm{W}$ peak, which further improves the sensitivity of the search. In order to avoid strange topologies with high $p_{\mathrm{T}}^{\text {miss }}$ and low $H_{\mathrm{T}}$, or vice-versa, a simultaneous selection on both quantities is possible by defining: $C_{\mathrm{T} 1} \equiv \min \left(p_{\mathrm{T}}^{\mathrm{miss}}, H_{\mathrm{T}}-100 \mathrm{GeV}\right)$ and $C_{\mathrm{T} 2} \equiv \min \left(p_{\mathrm{T}}^{\text {miss }}, p_{\mathrm{T}}(\mathrm{ISR})-25 \mathrm{GeV}\right)$, where the subscript number corresponds to the SR the requirement is applied in; for SR2, a selection on the ISR jet $p_{\mathrm{T}}$ is more effective than $H_{\mathrm{T}}$. Both SRs are split into two bins of $C_{\mathrm{T}}: 300<C_{\mathrm{T}}<400 \mathrm{GeV}$ and $C_{\mathrm{T}}>400 \mathrm{GeV}$.

\subsection{Multivariate Analysis (MVA) Method}

The multivariate analysis uses boosted decision trees (BDTs) [5] to take advantage of the correlations among observables, which are sometimes not obvious in a sequential selection optimisation. The set of input variables to the BDT consists of kinematic variables $p_{\mathrm{T}}^{\text {miss }}$ and $H_{\mathrm{T}}$, leptonic variables $p_{\mathrm{T}}(\ell), \eta(\ell), Q(\ell), m_{\mathrm{T}}$, jet variables $p_{\mathrm{T}}(\mathrm{ISR}), p_{\mathrm{T}}(\mathrm{b}), N_{\text {jets }}$, as well as quantities that take advantage of b-tagging algorithms $N\left(\mathrm{~b}^{\text {loose }}\right), \Delta R(\ell, \mathrm{b})$ and b-tagging discriminant $D(\mathrm{~b})$. The BDT is trained using simulated samples of the signal model with direct four-body decays, and $W+$ jets, $\bar{t}$ and $Z(\rightarrow v v)+$ jets events for the background. Due to the large dependence of the event kinematics on the $\Delta m$, each training is done as a function of the $\Delta m$, averaging over the $\tilde{t}$ masses. The resulting BDT output response for two extreme $\Delta m$ points in both data and simulation can be seen in Figure 3, where the differences in the distributions reflect the dependency of the signal event kinematics on the $\Delta m$ and BDT training optimisation. The SRs are defined by requiring a minimum value on the BDT discriminant, $B D T>X$, for each $\Delta m$ point, presented in Table 1.

\footnotetext{
${ }^{1}$ These updated plots have been taken from the corresponding paper [3], which has been submitted to JHEP.
} 

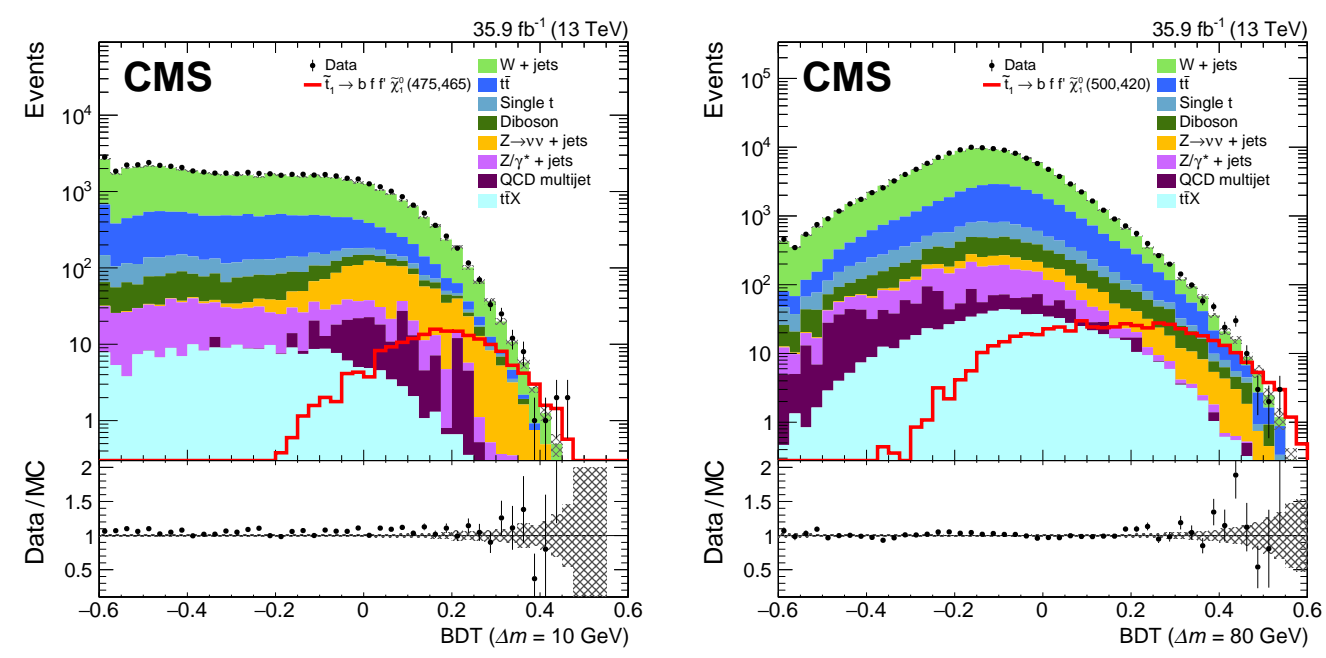

Figure 3: Distributions of the BDT output at the preselection level in data and simulation for $\Delta m$ of $10 \mathrm{GeV}$ (left) and $80 \mathrm{GeV}$ (right) [3].

\section{Background Estimation Methods}

The estimation of the dominant backgrounds in the analysis is done with a combined approach, using both data and MC simulation. Control regions (CRs) are designed such that they are dominated by the relevant backgrounds, from which one can make a precise extrapolation to the SRs. Background components with prompt and nonprompt leptons in the final state are estimated separately with dedicated methods.

The dominant prompt backgrounds originate from the $W+$ jets and $t \bar{t}$ processes. In the $\mathrm{C} \& \mathrm{C}$ approach, CRs are defined by inverting the lepton $p_{\mathrm{T}}$ selection, explicitly, $p_{\mathrm{T}}>30 \mathrm{GeV}$. Data/MC scale factors (SFs) are determined in these CRs and then applied to the simulation in the SRs in order to give the estimate of the background contribution. In other words, the data-MC scaling is extrapolated from a high $p_{\mathrm{T}}$ to a low $p_{\mathrm{T}}$ region. For the MVA approach, the prompt backgrounds are estimated directly from data in a CR defined by $B D T<0.2$.

The less dominant nonprompt lepton backgrounds, originating from QCD-multijet and Z decays to neutrinos $(Z(\rightarrow v v)+$ jets $)$, are estimated using the tight-to-loose method. This method relies on a 'loose' object definition, obtained by relaxing the lepton isolation and impact parameter $\left(d_{\mathrm{xy}}, d_{\mathrm{z}}\right)$ requirements, which, by construction, enriches the sample in nonprompt leptons. The 'tight' definition corresponds to the actual object definition used in the analysis. The probability that a nonprompt lepton passes both the tight and loose criteria (given it has already passed the loose), namely, the tight-to-loose ratio $\varepsilon_{\mathrm{TL}}$, is then determined in a QCD-enriched measurement region (MR), as a function of the lepton $p_{\mathrm{T}}$ and $\eta$. This is done using events from an orthogonal data set requiring very high $H_{\mathrm{T}}$, as well as low $p_{\mathrm{T}}^{\text {miss }}$ and $m_{\mathrm{T}}$ to reduce the contamination from prompt leptons. Application regions (ARs) are defined by loosening the leptonic cuts in the SRs and the events in these regions are reweighted by the $\varepsilon_{\mathrm{TL}}$ ratio to determine the expected nonprompt contribution in the SRs.

The remaining sub-leading backgrounds are estimated directly from simulation. 


\section{Results and Interpretation}

Several sources of background and signal systematic uncertainties are taken into account, such as the MC modelling of $p_{\mathrm{T}}$ and jet multiplicity distributions in $W+$ jets and $\bar{t} \bar{t}$ samples, pileup dependence, jet energy scale and resolution, object efficiencies or the luminosity uncertainty. The predicted yields in the SRs are compared to the observed data yields, as shown in Figure 4 and Table 1, for the C\&C and MVA approaches, respectively. The results are consistent with the SM expectations, as no significant deviation is observed.

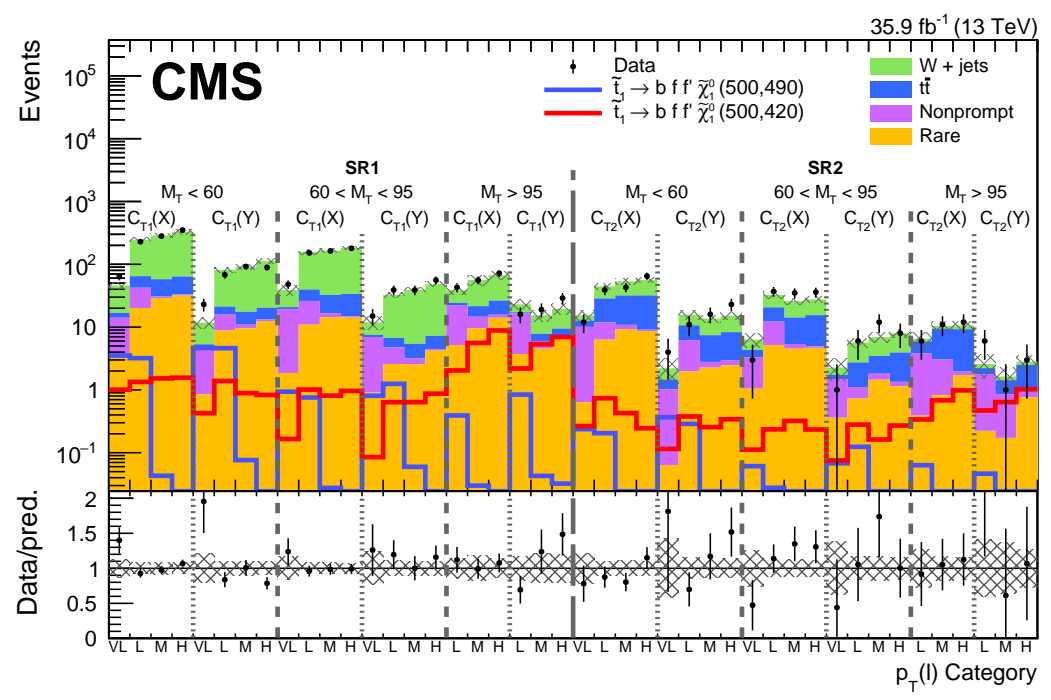

Figure 4: Summary of observed data and expected background yields in the C\&C SRs [3].

\begin{tabular}{l|c|cccc|c|c}
\hline \hline & BDT $>$ & $\begin{array}{c}Y_{\text {prompt }}^{\mathrm{SR}} \\
(W+\text { jets })\end{array}$ & $\begin{array}{c}Y_{\text {prompt }}^{\mathrm{SR}} \\
(\mathrm{t \mathfrak {t }})\end{array}$ & $Y_{\text {nonprompt }}^{\mathrm{SR}}$ & $\begin{array}{c}N^{\mathrm{SR}} \\
(\text { Rare })\end{array}$ & $N^{\mathrm{SR}}(\mathrm{B})$ & $N^{\mathrm{SR}}(\mathrm{D})$ \\
\hline$\Delta m=10 \mathrm{GeV}$ & 0.31 & $18.4 \pm 3.6$ & $1.8 \pm 4.8$ & $8.0 \pm 2.9$ & $2.3 \pm 1.4$ & $30.3 \pm 6.7$ & 39 \\
$\Delta m=20 \mathrm{GeV}$ & 0.39 & $9.0 \pm 2.0$ & $1.3 \pm 1.7$ & $11.2 \pm 3.2$ & $3.1 \pm 1.9$ & $24.7 \pm 4.5$ & 20 \\
$\Delta m=30 \mathrm{GeV}$ & 0.47 & $4.0 \pm 2.5$ & $1.2 \pm 0.6$ & $8.8 \pm 2.5$ & $1.7 \pm 1.2$ & $15.7 \pm 3.7$ & 22 \\
$\Delta m=40 \mathrm{GeV}$ & 0.48 & $4.1 \pm 1.3$ & $1.8 \pm 0.7$ & $7.6 \pm 2.3$ & $1.2 \pm 0.9$ & $14.8 \pm 2.8$ & 16 \\
$\Delta m=50 \mathrm{GeV}$ & 0.45 & $7.3 \pm 2.1$ & $4.7 \pm 2.8$ & $7.1 \pm 2.0$ & $5.5 \pm 3.1$ & $24.5 \pm 4.8$ & 36 \\
$\Delta m=60 \mathrm{GeV}$ & 0.50 & $2.0 \pm 0.6$ & $2.4 \pm 1.2$ & $3.1 \pm 1.1$ & $1.1 \pm 0.9$ & $8.7 \pm 1.8$ & 12 \\
$\Delta m=70 \mathrm{GeV}$ & 0.46 & $4.9 \pm 1.6$ & $3.4 \pm 1.1$ & $5.4 \pm 1.6$ & $3.2 \pm 1.9$ & $16.8 \pm 2.9$ & 20 \\
$\Delta m=80 \mathrm{GeV}$ & 0.44 & $7.1 \pm 1.6$ & $5.1 \pm 0.9$ & $5.3 \pm 1.6$ & $5.2 \pm 3.0$ & $22.8 \pm 3.3$ & 26 \\
\hline \hline
\end{tabular}

Table 1: Summary of observed data and expected background yields in the MVA SRs [3].

Upper limits are set on the top squark pair production cross section at 95\% CL in the plane of the top squark vs. LSP masses, excluding stop masses up to $560 \mathrm{GeV}$. The models assume a $100 \%$ branching ratio for the chosen decay channel; the decays are assumed to be prompt. The results are interpreted in terms of the four-body decay scenario, as shown in the two top diagrams of Figure 5. Furthermore, for the $\mathrm{C} \& \mathrm{C}$ approach, the results are interpreted in terms of chargino-mediated decay model, as seen at the bottom plot of Figure 5. Additionally, a statistical combination (black) of the $\mathrm{C} \& \mathrm{C}$ (blue) with the results of an all-hadronic analysis [6] (red) is presented, yielding the most stringent limits in this region of the SUSY parameter space with 2016 data. 

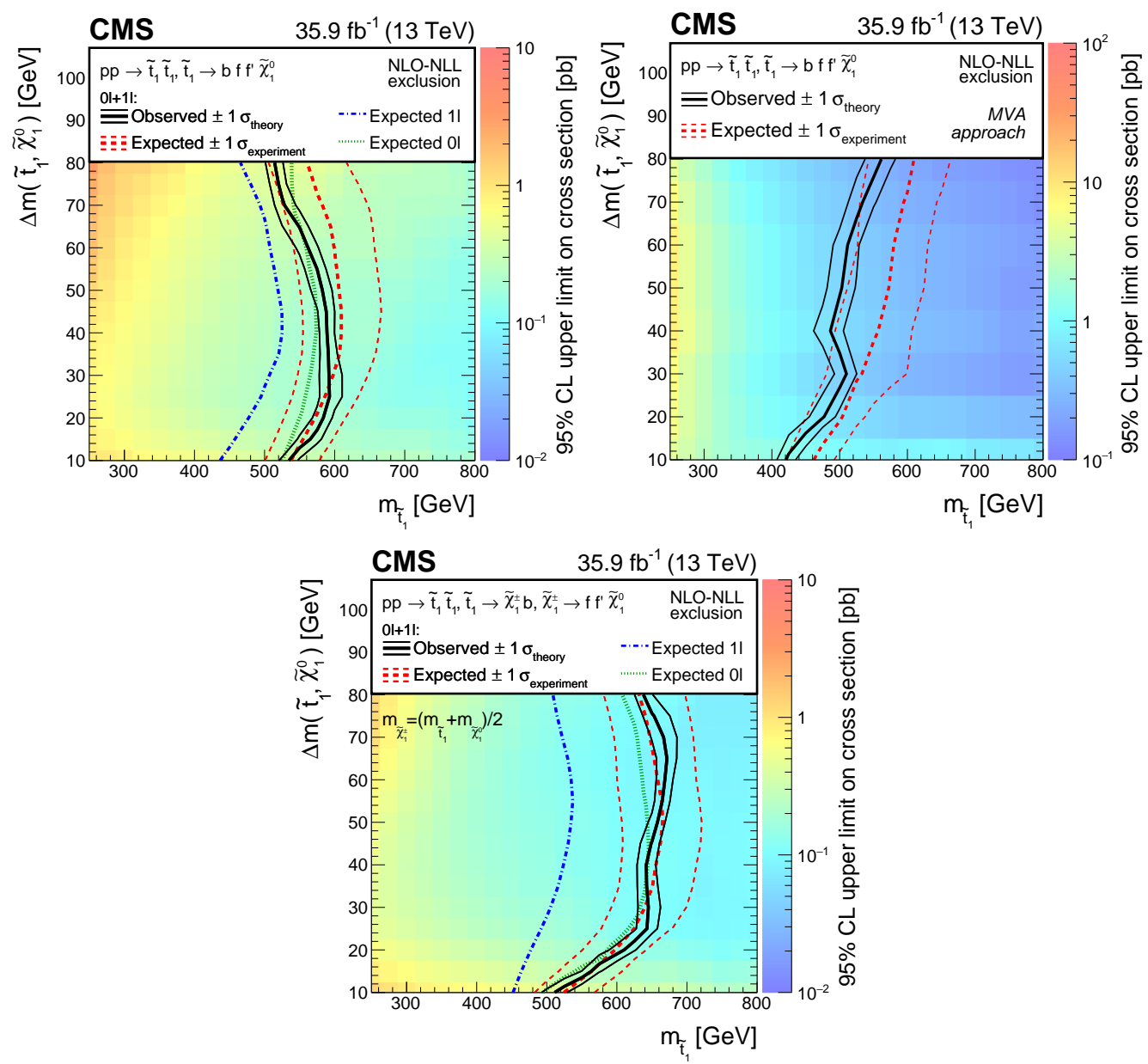

Figure 5: Limits on four-body stop decays (top) for the C\&C (left) and MVA (right) analyses. For the C\&C approach, limits are additionally set on chargino-mediated decays (bottom) [3].

\section{References}

[1] CMS Collaboration, The CMS Experiment at the CERN LHC, JINST 3 (2008) S08004.

[2] C. Balázs, M. Carena and C. E. M. Wagner, Dark matter, light stops and electroweak baryogenesis, Phys. Rev. D 70 (2004) 015007, [hep-ph/ 0403224 ].

[3] CMS Collaboration, Search for top squarks decaying via four-body or chargino-mediated modes in single-lepton final states in proton-proton collisions at $\sqrt{s}=13 \mathrm{TeV}$, Submitted to JHEP (2018), [1805.05784].

[4] M. Zarucki, Search for Supersymmetry with a Highly Compressed Mass Spectrum in the Single Soft Lepton Channel with the CMS Experiment at the LHC, PoS EPS-HEP2017 (2017) 728.

[5] L. Rokach and O. Maimon, Data mining with decision trees: theory and applications. World Scientific Pub Co Inc., 2008.

[6] CMS Collaboration, Search for direct production of supersymmetric partners of the top quark in the all-jets final state in proton-proton collisions at $\sqrt{s}=13 \mathrm{TeV}$, JHEP 10 (2017) 005, [1707. 03316]. 